\title{
Chiari malformation type 1: are we doing less with more? Illustrative case
}

\author{
Giuseppe Talamonti, MD, Erika Ferrari, MD, and Giuseppe D'Aliberti, MD \\ Department of Neurosurgery, Territorial Health and Social Services Authority Niguarda, Milan, Italy
}

\begin{abstract}
BACKGROUND Classic treatment of Chiari malformation type 1 consists of foramen magnum decompression. Selected patients may require occipitocervical fixation, transoral odontoidectomy, tonsillectomy, and so forth. Treatment standardization does not yet exist, and some patients risk being overtreated.

OBSERVATIONS A 20-year-old man with headache and Chiari malformation type 1 underwent extradural bone decompression. One year later, he was managed with the extradural section of his filum terminale. Eighteen months later, the patient underwent monitoring of intracranial pressure, occipitocervical stabilization, transoral odontoidectomy, minimally invasive subpial tonsillectomy, and occipital cranioplasty. His headache never changed, and he progressively developed hemiparesis and swallowing and respiratory disturbances. Two years later, a new magnetic resonance imaging scan showed extended syringomyelia with scarce peritonsillar subarachnoid space. The umpteenth operation consisted of the removal of a constricting epidural scar, arachnoid dissection, total tonsillectomy, creation of a wide subarachnoid space, and dural sac augmentation. The patient's initial postoperative course was smooth, and his headache improved. However, 8 days after surgery, the patient acutely presented with vegetative disturbances and died because of malignant brainstem edema of unknown origin.
\end{abstract}

LESSONS The story of this patient is not so uncommon. He underwent all the possible surgical treatments rather than a timely adequate osteodural decompression. Probably, he received less with more.

https://thejns.org/doi/abs/10.3171/CASE20145

KEYWORDS Chiari malformation; foramen magnum decompression; occipitocervical stabilization; transoral odontoidectomy; syringomyelia

Chiari malformation type 1 (CM1) is characterized by downward displacement of the cerebellar tonsils beneath the foramen magnum into the cervical spinal canal. The ectopic tonsils may impede the pulsation of the cerebrospinal fluid (CSF) and its circulation between the cranial and spinal compartments. ${ }^{1}$ Accordingly, the perimedullary CSF space may remain isolated and unable to dissipate the normal pressure increase that occurs during systole, breathing, Valsalva maneuvers, struggling, and changes of head position. ${ }^{1}$ This creates the conditions for the development of CM1 symptoms and a syrinx. Classic treatment of CM1 aims to achieve foramen magnum decompression (FMD) and to restore the CSF flow. ${ }^{1}$ The FMD consists of occipital craniectomy and $\mathrm{C} 1$ laminectomy and can comprise bone-only decompression, dural opening (with or without duraplasty), arachnoid dissection (with or without exploration of the Magendie foramen), and tonsillectomy. Selected cases may require occipitocervical fixation with or without transoral odontoidectomy.
Modern techniques and skillfulness are so advanced that renowned and honorable specialists sometimes get carried away by their own dexterity and perform surgeries that may not be strictly necessary. Furthermore, there are unrecognized and nonvalidated treatments (such as the extradural section of the filum terminale) that are proposed on the pretext of being noninvasive. As a consequence, an increasing number of patients are undergoing multiple procedures that may prove to be of little use. In this paper, we report a case study that merits further discussion.

\section{Illustrative Case}

A 20-year-old man was admitted to a local hospital because of a 1-month history of headache. Magnetic resonance imaging (MRI) showed ectopic cerebellar tonsils at the $\mathrm{C} 1$ level. The patient underwent extradural bony decompression with partial-thickness durotomy. Postoperatively, CSF leakage occurred, and reoperation was necessary to repair the dura using stitches and fibrin glue.

ABBREVIATIONS $\mathrm{CM} 1=$ Chiari malformation type $1 ; \mathrm{CSF}=$ cerebrospinal fluid; $\mathrm{CT}=$ computed tomography; $\mathrm{FMD}=$ foramen magnum decompression; $\mathrm{ICP}=$ intracranial pressure; $M R I=$ magnetic resonance imaging.

INCLUDE WHEN CITING Published February 8, 2021; DOI: 10.3171/CASE20145.

SUBMITTED December 8, 2020. ACCEPTED December 28, 2020.

(c) 2021 The authors, CC BY-NC-ND 4.0 (http://creativecommons.org/licenses/by-nc-nd/4.0/) 
The patient's headache did not improve. One year later, the patient was referred to a private center abroad, where an extradural section of the filum terminale was performed, although there was no evidence of a tethered cord. This had no effect on his headache.

Eighteen months later, an MRI scan showed a small syringobulbia with normal cerebral ventricles (Fig. 1 left). The patient was then admitted to a specialized hospital. First, he underwent prolonged intracranial pressure (ICP) monitoring that showed normal patterns. Afterward, despite the absence of any craniocervical hypermobility, basilar impression, and platybasia (Fig. 1 right), he underwent craniocervical fixation during cervical traction (using an artificial bone substitute and screws on the condyles, C1, and C2) (Fig. 2) and transoral odontoid removal. Finally, minimally invasive subpial tonsillectomy and occipital cranioplasty were performed.

In the following 2 years, the patient progressively experienced nocturnal apnea, swallowing disturbances, right hemiparesis, and invalidating nuchal/neck pain. He was then admitted to our hospital. Another MRI scan showed extended syringomyelia and reduced subarachnoid spaces, with only a scarce possibility of CSF flow (Fig. 3 left).

The fixed head position was perceived by the patient as intolerable and was considered to be the cause of his neck pain and swallowing/ respiratory difficulties. The screwing and bone substitute precluded any surgical modification of the head position (Fig. 2).

The umpteenth operation consisted of the removal of constricting thick and hard epidural scars, complete tonsillectomy, extended subarachnoid dissection with the creation of a wide subarachnoid space from the 4th ventricle to the perimedullary space, and dural sac augmentation. The initial postoperative period was uneventful. Postoperatively, the patient's headache improved, and a further MRI scan showed initial shrinkage of the syringobulbia (Fig. 3, right).

Eight days after surgery, the patient experienced violent nuchal pain with abrupt wound swelling and bleeding. Moderate arterial hypertension and tachycardia were found. An acute subgaleal clot due to the spontaneous laceration of the occipital artery was removed. The epidural space was explored, but no further clot was found. The
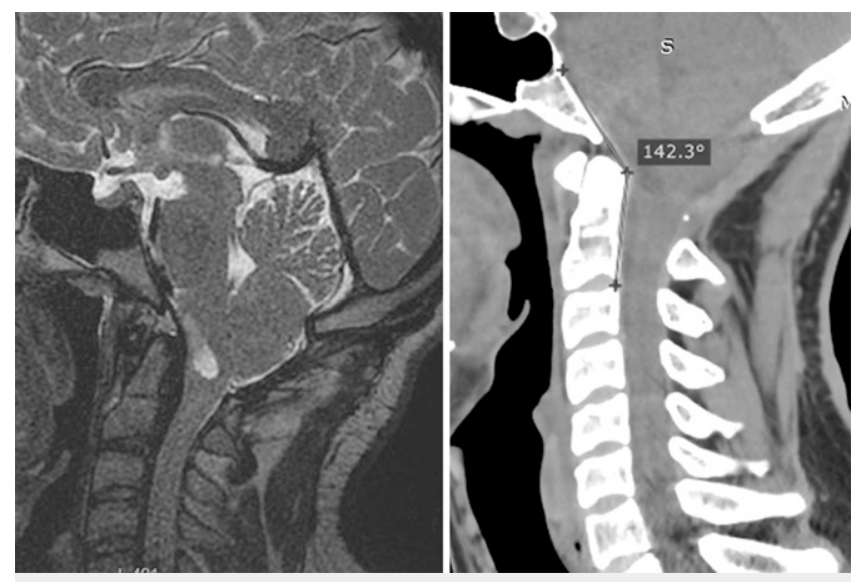

FIG. 1. Left: MRI (sagittal view) performed 30 months after extradural bone decompression. A small syrinx was evident. There were scarce subarachnoid spaces. The cerebral ventricles were normal, and there was no evidence of pannus at the odontoid level. Right: CT scan (sagittal reconstruction) showing a normal clivoaxial angle $\left(142^{\circ}\right)$. The Grabb-Oakes and Harris measurements were also normal.
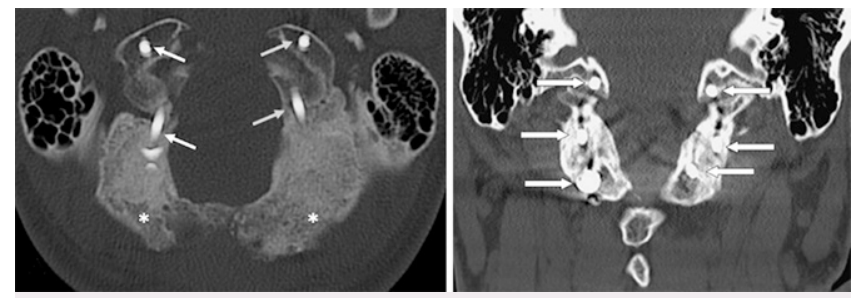

FIG. 2. CT scans. Axial view (left) and coronal reconstruction (right) 2 years after craniocervical fixation. The screws (arrows) were embedded in abundant artificial bone substitute (asterisks), which was also used for cranioplasty. Any attempt at surgical modification of the head position was deemed impossible.

patient's recovery from the anesthesia was slow, but no new neurological focality was evident. A computed tomography (CT) scan, a CT angiogram, and an MRI scan were clear apart from some signs of mild brainstem edema. The findings of blood test assays and vegetative parameters were normal. The patient was admitted to the neurosurgical intensive care unit, but then he developed malignant brainstem edema. His ICP was normal and only increased in the terminal phase. He died soon after. His parents refused autopsy.

\section{Discussion}

FMD is effective in approximately $80 \%$ of cases, provided that indications and techniques are correct. ${ }^{1-3}$ Patients who do not obtain benefit have been known to visit different centers in search of alternative (sometimes debatable) therapies. These unsatisfied patients often undertake pilgrimages to different clinics until they find someone who promises a miracle. We suspect that treatment billability may

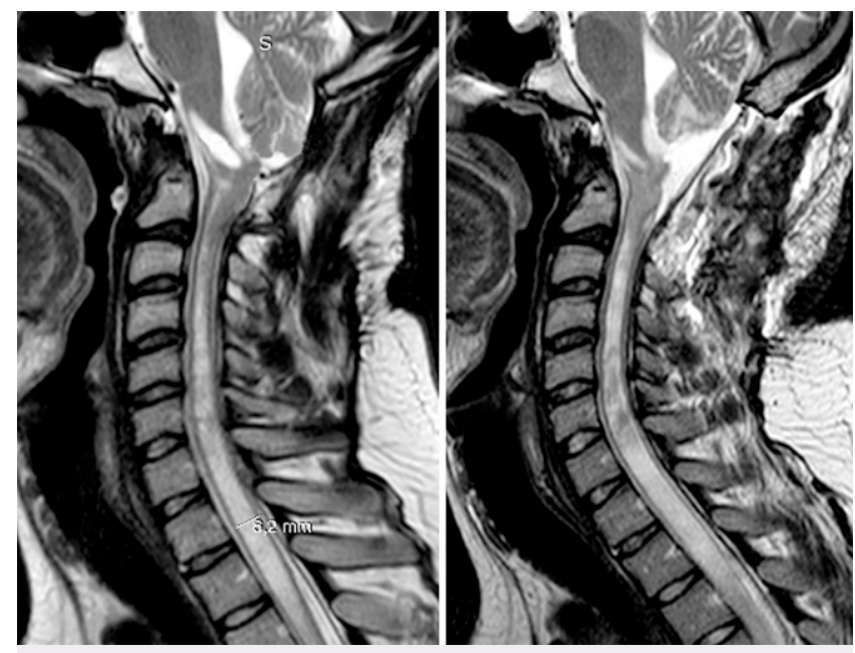

FIG. 3. MRI scans (sagittal view). Left: Image obtained 2 years after craniocervical fixation, odontoid removal, and minimally invasive subpial tonsillectomy. The syringobulbia was grossly unchanged, but extended syringomyelia had developed. The posterior subarachnoid space was virtually absent, whereas a thin ventral film was visible. Right: Image obtained 3 days after tonsillectomy, arachnoid dissection, and dural augmentation. The syringobulbia had decreased, whereas the syringomyelia was unchanged. A wide subarachnoid space was freely communicating with the 4th ventricle. 
become crucial. Apart from the outcome, our patient's history is not an isolated example and has led to some reflection on our part.

\section{Observations}

Initial Indication

The incidence of CM1 is estimated as $<1 \%$, but an increasing number of ectopic cerebellar tonsils are now discovered because of the diffusion of MRI. 2,4,5 There is general agreement that surgery should be reserved for symptomatic patients, ${ }^{4,6}$ but the correct assessment of preoperative symptoms may not be simple. For instance, headache is very frequent in patients with $\mathrm{CM} 1$, but the differential diagnosis of headache may be quite difficult. Wrong indication for surgery represents one of the main causes of unsatisfactory results. ${ }^{6,7}$ Because patients with $\mathrm{CM} 1$ very rarely need emergency treatment, ${ }^{8}$ a wait-andsee attitude is reasonable in all doubtful cases. Treatment is sometimes indicated, however, in patients with $\mathrm{CM}$ who could just be observed. The fact is that FMD is often considered a simple procedure that does not require particular surgical skillfulness.

The reported patient was surgically managed because an MRI scan had shown ectopic tonsils during a screening for persisting headache. We are not sure this patient needed any surgery at that time. A more prudent attitude would have been reasonable.

\section{Extradural Bone Decompression}

Currently, limited occipital craniectomy is recommended to prevent several postoperative complications, including cerebellar ptosis. In the reported case, the initial occipital craniectomy was relatively large and required a cranioplasty during one of the subsequent operations. This probably played no role in the patient's clinical history but should have been avoided.

Bone-only decompression has been proposed to minimize the surgical risks, particularly to prevent CSF leaks, which, however, may occur anyway. Partial-thickness durotomy is a rational option in children, in whom the dura may be easily weakened. In adults, dural delamination is more difficult, and better results have been reported with dural augmentation. ${ }^{1}$

The present patient experienced postoperative CSF leakage that required dural repair by means of fibrin glue. This could have played a role in the development of arachnoid adherences and subsequent complications. Basically, a possibly unjustified operation might have made the evolution worse.

In patients with $\mathrm{CMI}$, both indications and surgical treatment may not be as simple as one might think. FMD may present unexpected pitfalls, and the CSF leakage tends to be more frequent than in other posterior fossa procedures, probably because of the chronic alteration of the CSF dynamics. Accordingly, the FMD should not be delegated to surgeons with poor experience in the management of patients with $\mathrm{CMI}$.

\section{Section of the Filum Terminale}

An inviolable principle states that doctors must offer their patients what they believe to be the best possible treatment. Unrecognized or experimental treatments may be proposed in selected cases, but they should be complementary. The correlation between CM1 and "occult tethered cord syndrome" has been postulated but is very rare..$^{9,10}$ In these exceptional cases, a detethering procedure may be indicated to address the tethered cord syndrome, but it does not produce effects on CM1. ${ }^{11}$

Nowadays, clever advertising campaigns may convince patients with $\mathrm{CM} 1$ to pay for a treatment that lacks anatomical foundations (extradural section of the filum terminale makes no sense), that is devoid of pathophysiological justification (these patients do not present with tethered cords), and that is not supported by clinical evidence (no reliable series have been published). ${ }^{10}$ We therefore think that international scientific societies should take a definite position on this matter. In particular, we hope that someone can find a means of dealing with those who propose this treatment to each patient with CM1.

\section{Occipitocervical Fixation and Odontoidectomy}

Craniocervical instability has been reported in approximately $10 \%$ of patients with $\mathrm{CM} 1$, usually in association with collagenopathies. ${ }^{12}$ Recently, a pathogenetic role of instability has even been postulated..$^{13}$ Particularly controversial is fixation, which has been proposed as the treatment of choice for patients with $\mathrm{CM} 1$ without instability or craniocervical joint anomalies. ${ }^{14}$ The indication for craniocervical fixation needs careful reflection. Instability is never easy to demonstrate radiologically, even using dynamic three-dimensional CT scans. Moreover, fixation remains an invasive and relatively risky procedure, even in the most skilled hands. ${ }^{15}$ According to AO Spine, craniocervical fixation per se must be considered a major morbidity. Overall patient dissatisfaction, persistent pain, and swallowing and respiratory difficulties are probably underreported. ${ }^{16}$ The patient in question underwent condylar screwing and arthrodesis using abundant bone substitute. This represented an elegant and powerful method of stabilization, but subsequent adjustments were impossible, and the head position could be not corrected. Perhaps other types of fixation would have been preferable.

Transoral odontoidectomy is mandatory in cases of symptomatic true basilar invagination and is recommended in platybasia, when the clivoaxial angle is $<135^{\circ} .{ }^{17,18}$ However, the data cited should be treated with caution; all series include patients with modest basilar impression or some degree of platybasia and with no significant brainstem compression. ${ }^{17,18}$ The treatment of these alterations is necessary in a minority of patients with $\mathrm{CM} 1$, and most of the authors recommend simple traction/fixation rather than transoral decompression. ${ }^{3,17,18} \mathrm{~A}$ reasonable option is to attempt a posterior reduction first and, in case of failure, to perform transoral odontoidectomy and posterior fusion. This would allow the surgeon to treat microinstability (if really present) and to avoid the anterior decompression in a significant number of patients whose odontoid is reducible.

We see that a worrisome trend to propose prophylactic surgery is emerging in clinical practice. We are of the view that prolonged ICP monitoring, craniocervical fixation, and transoral odontoidectomy were of debatable value in the presented patient. MRI showed limited subarachnoid space, but just a minimally invasive subpial tonsillectomy was performed. This was an elegant technique too, but this particular patient probably needed something more.

\section{Restoration of Intracranial-Extracranial CSF Communication}

FMD remains the mainstay of surgical treatment for $\mathrm{CM} 1 .^{1,3}$ The reported patient had scarce subarachnoid space with presumably impaired CSF flow. Nevertheless, the previous treatments mainly addressed other issues. We aimed to restore the normal flow of the CSF through the foramen magnum, but the consequences were ominous. We wonder what really happened in the present case. Sudden spontaneous bleeding from a subgaleal artery is unusual 8 days after surgery, and we suppose it depended on arterial hypertension that was triggered by an alteration of the bulbar nuclei. The subgaleal clot did not produce parenchymal compression. Accordingly, we think that it was the consequence and not the cause of the patient's brainstem 
dysfunction. Apart from initial mild edema, neuroimaging showed neither vascular nor parenchymal lesions, and bulbar-cerebellar ptosis could be excluded. The patient's ICP remained normal almost to the very end, when repeated MRI showed malignant edema. Autopsy could not be performed, but we doubt that it would have added anything to the findings of the CT scan, CT angiogram, and MRI scan.

To our mind, when the syrinx developed, adequate cerebellobulbar decompression would probably have been preferable; instead, other strategies were chosen. The long history of compression and altered CSF circulation, as well as the multiple ineffective operations, led to chronic brainstem impairment. After the last operation, MRI showed initial syrinx shrinkage, but we suppose that the impaired brainstem was unable to tolerate the new situation. Perhaps the last surgery simply decompensated a chronically delicate and unstable equilibrium.

\section{Lessons}

Although CM1 and FMD may appear uncomplicated, substantial experience is needed to assess the correct indications and to choose the proper techniques. Several therapeutic options exist, and, of course, the best surgical treatment is that tailored to the characteristics of each patient. Selected patients may require particular treatments, but the therapeutic choices must be carefully pondered. The ability to perform a complex procedure does not justify its use in all cases. There is an old saying: "If you have a hammer, you will see nails everywhere."

In our practice, we often encounter patients with CM1 to whom someone has offered other (sometimes complex) treatments instead of an adequate FMD. We need to avoid this trend toward "doing less with more."

\section{Acknowledgments}

We thank the patient associations that support and advise their members throughout their complex diagnostic/therapeutic process.

\section{References}

1. Oldfield EH. Pathogenesis of Chiari I - pathophysiology of syringomyelia: implications for therapy: a summary of 3 decades of clinical research. Neurosurgery. 2017;64(CN suppl 1):66-77.

2. Milhorat TH, Chou MW, Trinidad EM, et al. Chiari I malformation redefined: clinical and radiographic findings for 364 symptomatic patients. Neurosurgery. 1999;44(5):1005-1017.

3. Klekamp J. Chiari I malformation with and without basilar invagination: a comparative study. Neurosurg Focus. 2015;38(4): E12.

4. Meadows J, Kraut M, Guarnieri M, et al. Asymptomatic Chiari type I malformations identified on magnetic resonance imaging. J Neurosurg. 2000;92(6):920-926.

5. Menezes AH, Greenlee JD, Donovan KA. Honored guest presentation: lifetime experiences and where we are going: Chiari I with syringohydromyelia-controversies and development of decision trees. Clin Neurosurg. 2005;52:297-305.

6. Novegno F. Clinical diagnosis-part II: what is attributed to Chiari I. Childs Nerv Syst. 2019;35(10):1681-1693.

7. Ciaramitaro P, Ferraris M, Massaro F, et al. Clinical diagnosis_-partl: what is really caused by Chiari I. Childs Nerv Syst. 2019;35(10):1673-1679.
8. Talamonti G, Marcati E, Gribaudi G, et al. Acute presentation of Chiari 1 malformation in children. Childs Nerv Syst. 2020;36(5): 899-909.

9. Canheu AC, Santos MV, Furlanetti LL, et al. The Brazilian Society for Pediatric Neurosurgery: consensus on Chiari I deformity. Childs Nerv Syst. 2020;36(1):17-18

10. Massimi L, Peraio S, Peppucci E, et al. Section of the filum terminale: is it worthwhile in Chiari type I malformation? Neurol Sci. 2011;32(suppl 3):S349-S351.

11. Glenn C, Cheema AA, Safavi-Abbasi S, et al. Spinal cord detethering in children with tethered cord syndrome and Chiari type 1 malformations. J Clin Neurosci. 2015;22(11):1749-1752.

12. Milhorat TH, Bolognese PA, Nishikawa M, et al. Syndrome of occipitoatlantoaxial hypermobility, cranial settling, and Chiari malformation type I in patients with hereditary disorders of connective tissue. J Neurosurg Spine. 2007;7(6):601-609.

13. Goel A. Is atlantoaxial instability the cause of Chiari malformation? Outcome analysis of 65 patients treated by atlantoaxial fixation. J Neurosurg Spine. 2015;22(2):116-127.

14. Goel A, Kaswa A, Shah A. Atlantoaxial fixation for treatment of Chiari formation and syringomyelia with no craniovertebral bone anomaly: report of an experience with 57 cases. Acta Neurochir Suppl (Wien). 2019;125:101-110.

15. Lall R, Patel NJ, Resnick DK. A review of complications associated with craniocervical fusion surgery. Neurosurgery. 2010; 67(5):1396-1403.

16. Bagley CA, Witham TF, Pindrik JA, et al. Assuring optimal physiologic craniocervical alignment and avoidance of swallowingrelated complications after occipitocervical fusion by preoperative halo vest placement. J Spinal Disord Tech. 2009;22(3): 170-176.

17. Henderson FC Sr, Henderson FC Jr, Wilson WA 4th, et al. Utility of the clivo-axial angle in assessing brainstem deformity: pilot study and literature review. Neurosurg Rev. 2018;41(1):149-163.

18. Kubota M, Yamauchi T, Saeki N, et al. Surgical results of foramen magnum decompression for Chiari type 1 malformation associated with syringomyelia: a retrospective study on neuroradiological characters influencing shrinkage of syringes. Spinal Surg. 2004 18:81-86.

\section{Disclosures}

The authors report no conflict of interest concerning the materials or methods used in this study or the findings specified in this paper.

\section{Author Contributions}

Conception and design: Talamonti. Acquisition of data: Talamonti, Ferrari. Analysis and interpretation of data: Talamonti, D'Aliberti. Drafting the article: Talamonti. Critically revising the article: Talamonti, Ferrari. Reviewed submitted version of manuscript: Talamonti, Ferrari. Approved the final version of the manuscript on behalf of all authors: Talamonti.

\section{Correspondence}

Giuseppe Talamonti: Niguarda Ca'Granda Hospital, Milan, Italy. tala_nch@yahoo.it; giuseppe.talamonti@ospedaleniguarda.it. 